\title{
Topological skeleton of the 2-D slightly non-ideal MHD system close to $X$-type magnetic null points - an analysis of the general solution for the generic case
}

\author{
D. H. Nickeler ${ }^{1}$, M. Karlický ${ }^{1}$, and M. Bárta ${ }^{1,2}$ \\ ${ }^{1}$ Astronomical Institute AV ČR Ondřejov, Fričova 298, 25165 Ondrejov, Czech Republic \\ ${ }^{2}$ Max-Planck-Institut für Sonnensystemforschung, Max-Planck-Strasse 2, 37191 Katlenburg-Lindau, Germany \\ Correspondence to: D. H. Nickeler (nickeler@ asu.cas.cz)
}

Received: 22 February 2011 - Revised: 23 May 2012 - Accepted: 6 June 2012 - Published: 29 June 2012

\begin{abstract}
The appearance of eruptive space plasma processes, e.g. in eruptive flares as observed in the solar atmosphere, is usually assumed to be caused by magnetic reconnection, often connected with singular points of the magnetic field.

We are interested in the general relation between the eigenvalues of the Jacobians of the plasma velocity and the magnetic field and their relation to the shape of a spatially varying, localized non-idealness or resistivity, i.e. we are searching for the general solution. We perform a local analysis of almost all regular, generic, structurally stable non-ideal or resistive MHD solutions. Therefore we use Taylor expansions of the magnetic field, the velocity field and all other physical quantities, including the non-idealness, and with the method of comparison of coefficients, the non-linear resistive MHD system is solved analytically, locally in a close vicinity of the null point.

We get a parameterised general solution that provides us with the topological and geometrical skeleton of the resistive MHD fields. These local solutions provide us with the "roots" of field and streamlines around the null points of basically all possible 2-D reconnection solutions. We prove mathematically that necessarily, the flow close to the magnetic X-point must also be of X-point type to guarantee positive dissipation of energy and annihilation of magnetic flux. We also prove that, if the non-idealness has only a onedimensional, sheet-like structure, only one separatrix line can be crossed by the plasma flow, similar to known reconnective annihilation solutions.
\end{abstract}

Keywords. Space plasma physics (Magnetic reconnection)

\section{Introduction}

Magnetic reconnection is thought to be a process responsible for many eruptive plasma phenomena in space plasmas and astrophysical plasmas, like geomagnetical substorms or solar flares. Although magnetic reconnection in two dimensions (2-D) is fairly well understood (e.g. see the comments in Baty et al., 2009), not much is known about the topological and geometrical structure of flow and field lines in the vicinity of the singular points of plasma flow and magnetic field.

The first reconnection scenarios by Petschek (1964) and Sweet-Parker (see, e.g. Sweet, 1958) proposed a magnetic null point and a stagnation point flow into the diffusion region, i.e. the stagnation point is inside this diffusion region.

Later, Priest and Cowley (1975) analysed the case of incompressible 2-D MHD with constant resistivity. They showed that either the magnetic field is of hyperbolic type ("X-type") and the flow is a shear flow, or the magnetic field is of higher order (and "sheared") and the flow has a typical shape of a stagnation point flow (hyperbolic). In the former case reconnection takes place, while the latter case results in so-called annihilation solutions.

Annihilation solutions have further been studied by Craig and Henton (1995) who chose a special ansatz for the solution of the resistive MHD to get reconnection solutions. They solved the first order momentum equation and the resistive Ohm's law, starting with a nonlinear perturbation of magnetic annihilation solutions. This lead them to so-called "reconnective annihilation" solutions, where only one of the two separatrix-lines is crossed, and the other is only tangent to the converging streamlines. The current sheet has a one-dimensional structure (straight line), while curvilinear 
current sheets were subsequently studied by Tassi et al. (2002) and Titov et al. (2004). The results found by Craig and Henton (1995) and Craig and Rickard (1994) confirm the results found earlier by Priest and Cowley (1975), who found more "shear-like" flows instead of typical stagnation point flows.

It was shown by Priest et al. (1994) and later on in extended form by Watson and Craig (1998) that under certain circumstances, like constant resistivity or current depending/anomalous resistivity and sub-Alfvénic flow, etc., reconnection is impossible: the so-called anti-reconnection theorems.

What is missing however in the framework of the 2-D resistive reconnection problematics is a detailed analysis on the relation between the stable topology of magnetic field and stream lines, and the topology and geometry of any nonconstant, i.e. spatially dependent resistivity. Therefore it restricts every possible solution of the slightly non-ideal MHD system Eqs. (9)-(15), i.e. in particular we derive a general solution for incompressible resistive MHD in the close vicinity of the null point of flow and field. We prove in the following that also "typical" stagnation point flows are possible ("Xtype"), and exclude here the "shear-like" flows.

\section{Assumptions and basic equations}

\subsection{The topological and geometrical structure of the magnetic field}

The topological classification of 2-D vector fields in the vicinity of their null points is described in the literature (with connection about phase portraits of dynamical systems, i.e. systems of ordinary differential equations) (e.g. of Arnold, 1992; Amann, 1995; or Reitmann, 1990). The topological structure of magnetic fields in the vicinity of null points is described, e.g. by Parnell et al. (1996) and concerning the construction of ideal MHD flows, e.g. in Nickeler et al. (2006).

Our interest is to ask which topology and geometry of the macroscopic flow correspond to which magnetic topology and geometry of the magnetic field in the frame of MHD. In contrast to the analyses mentioned in the previous paragraph, we here have to investigate the topological and geometrical properties of both vector fields, i.e. that of the plasma flow and that of the magnetic field. To perform this analysis we use a Taylor expansion of both fields in the close vicinity of their null points. As we are interested in the local structure of the fields only, we neglect all derivatives of the order higher than one. Such a treatment is justified because we are interested in how the field and streamlines are rooted in the null points of flow and field, represented by the eigenvalues of the Jacobians.

A similar approach for 3-D, but restriced to constant resistivity, was done by Titov and Hornig (2000). In 2-D the restriction to constant resistivity would lead to a field-aligned flow, i.e. without crossing the magnetic separatrices and, as we will see in the following (Sect. 2.2), to a vanishing electric field. Such a configuration does not allow for reconnection. In 3-D Titov and Hornig (2000) found additional non-field-aligned-flows, so-called SFC-flows (spiralfield-crossing flows). But as the resistivity was constant and also the current density, the flow can cross the field lines in the fan-plane, but not cross the fan-plane itself. Also, no flux transport in the vicinity of the nulls can take place as the nonidealness $\eta \boldsymbol{j}$ is constant. This interesting dynamic is therefore close to reconnection, but is not a "classical" reconnection scenario. In this investigation we will basically follow the method of Titov and Hornig (2000), but allow for a spatially varying resistivity.

Some detailed and important remarks concerning the local use of linear fields or better to say linearized fields are:

Morse-Smale systems are "typical" or generic vector fields on two-dimensional compact orientable manifolds. They have basically a non-vanishing real part of the eigenvalues at the null points, but also periodic orbits (in 2-D elliptic null point) with zero real part are allowed due to the divergencefree nature of magnetic fields (see, e.g. the theorem 15.1 in Reitmann, 1990). The inclusion of a guide field would change the typical character of a 2-D null point topology: the classical guide field is constant, and would therefore destroy the generic singular structure in the vicinity of the 2-D magnetic null point. If the guide field $B_{Z}$ depends on $x$ and $y$ and a magnetic null point should exist, then the situation would be basically the same in the close vicinity of the null point as with constant guide field. As an addition of a guide field would destroy the generic topological properties of the 2-D case, we will exclude a guide field within this investigation.

Every vector field on a manifold is basically equivalent to a dynamical system (see, e.g. Arnold, 1992). Dynamical Systems not having Morse-Smale structure in 2-D are topologically/structurally unstable and will not occur in nature, as any small perturbation will "destroy" such systems (see, e.g. Hornig and Schindler, 1996; Bruce and Giblin, 1992 or Reitmann, 1990; and for a mathematical proof Peixoto, 1959). Solutions, depending on singular perturbation theory, are therefore exceptions and thus, in a general sense, "unphysical". As almost all functions are of Morse-type, without loss of generality, we know that the (effective) resistivity is a quadratic form, like the flux function $A$ and the stream function $\psi$ (see Eqs. 3 and 34). Our approach, in contrast to the above mentioned, guarantees reasonable and structurally stable fields, being necessary to define magnetic reconnection or similar dynamics.

The topological and geometrical "roots" can be determined by an arbitrary contracting mapping such that only the lowest non-trivial orders remain. Then we can express the class of linear velocity fields with the help of their first derivatives, i.e. Jacobians: 


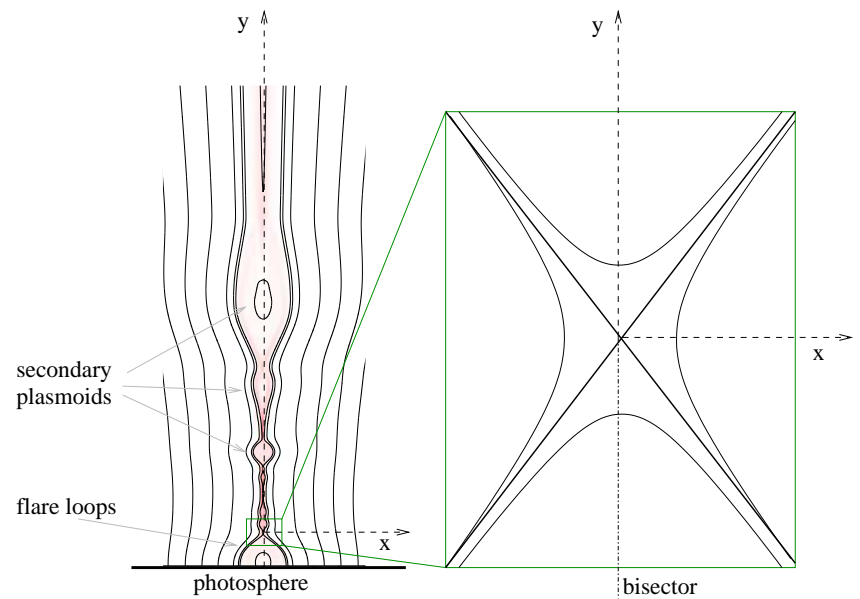

Fig. 1. Scenario of a flare loop with an X-point.

$\boldsymbol{v}=\stackrel{\leftrightarrow}{\boldsymbol{V}} \boldsymbol{x}=\left(\begin{array}{ll}V_{11} & V_{12} \\ V_{21} & V_{22}\end{array}\right)\left(\begin{array}{l}x \\ y\end{array}\right)$,

in analogy to the magnetic field $\boldsymbol{B}$

$\boldsymbol{B}=\stackrel{\leftrightarrow}{\boldsymbol{B}} \boldsymbol{x}=\left(\begin{array}{ll}B_{11} & B_{12} \\ B_{21} & B_{22}\end{array}\right)\left(\begin{array}{l}x \\ y\end{array}\right)$.

According to Parnell et al. (1996), every magnetic field of the form of Eq. (2) can be rotated such, that it is represented by a magnetic flux function $A=a x^{2}+b y^{2}$, so that a standard null point of the magnetic field appears with a constant current density $j_{\mathrm{z}}=j_{0}=$ const around the origin, where the magnetic field is given by

$\nabla A \times \boldsymbol{e}_{\mathrm{Z}}=\boldsymbol{B}$.

The corresponding scenario of a flare loop is given in Fig. 1.

The current density is given by

$\nabla \times \boldsymbol{B}=-\Delta A \boldsymbol{e}_{\mathrm{Z}}=\mu_{0} j_{\mathrm{Z}} \boldsymbol{e}_{\mathrm{Z}}$,

such that

$\boldsymbol{B}=\left(\begin{array}{cc}0 & 2 b \\ -2 a & 0\end{array}\right)\left(\begin{array}{l}x \\ y\end{array}\right) \equiv\left(\begin{array}{cc}0 & \frac{\mu_{0}}{2}\left(j_{\mathrm{t}}-j_{\mathrm{z}}\right) \\ \frac{\mu_{0}}{2}\left(j_{\mathrm{t}}+j_{\mathrm{z}}\right) & 0\end{array}\right)\left(\begin{array}{l}x \\ y\end{array}\right)$.

The different topologies of 2-D vector fields are represented by two independent parameters, the threshold current $j_{\mathrm{t}}$, and the eigenvalues of $\stackrel{\leftrightarrow}{B}$, the $\lambda_{B}$ 's, or $j_{\mathrm{z}}$, the current in $z-$ direction

$\lambda_{B}= \pm \frac{\mu_{0}}{2} \sqrt{j_{t}^{2}-j_{z}^{2}}= \pm(2 \sqrt{-a b})$,

implying a bijective relation between the variables $a$ and $b$ and the threshold $j_{\mathrm{t}}$ and the actual current $j_{\mathrm{z}}$, to be precise

$$
\begin{aligned}
a & =-\frac{\mu_{0}}{4}\left(j_{\mathrm{t}}+j_{\mathrm{z}}\right) \quad b=\frac{\mu_{0}}{4}\left(j_{\mathrm{t}}-j_{\mathrm{z}}\right) \\
\Leftrightarrow j_{\mathrm{z}} & =-\frac{2}{\mu_{0}}(a+b) \quad j_{\mathrm{t}}=\frac{2}{\mu_{0}}(b-a) .
\end{aligned}
$$

We will use the four variables $a, b, j_{\mathrm{t}}, j_{\mathrm{z}}$ if it is convenient, i.e. combinations of the four to simplify the corresponding terms in the equations.

The eigenvalue $\lambda_{B}$ determines the topological structure of the field and the geometrical shape of the field lines. For a divergence free case there are only three main types of such fields:

- the case that the eigenvalue is zero $\left(\left|j_{\mathrm{t}}\right|=\left|j_{\mathrm{z}}\right|\right)$ corresponds to a field with one-dimensional magnetic field lines (degenerated case), see Eq. (5), or a vanishing field

- the case that $\left|j_{\mathrm{t}}\right|<\left|j_{\mathrm{z}}\right|$ corresponds to field lines being topological circles (geometrical ellipses), if $j_{\mathrm{t}}=0$ ( $a=$ $b)$, then geometrical circles. All the cases mentioned in the last sentence are so-called O-points, and

- the case of the so-called X-points, where $\left|j_{\mathrm{t}}\right|>\left|j_{\mathrm{Z}}\right|$.

Only for the case in the last item a magnetic separatrix exists. Such separating field lines must exist to enable magnetic reconnection. A necessary condition for magnetic reconnection in 2-D is that the plasma flow crosses magnetic separatrices (see, e.g. Priest and Forbes, 2000; Vasyliunas, 1975; Cowley, 1976; Sonnerup, 1979; or Sonnerup et al., 1984). The current-free case is given by $j_{\mathrm{z}}=0$, i.e. $a=-b$ and should be excluded, as dissipation in such a case would have nothing to do with the electric current.

\subsection{The basic incompressible resistive MHD equations and assumptions}

The following analysis is restricted to pure incompressible and resistive dynamics, i.e. ohmic heating without any other loss terms like viscosity or heat conduction. The resistivity $\eta$ should be localised, obeying the boundary conditions. The basic non-ideal/resistive stationary MHD equations in 2-D are given by (following, e.g. Goedbloed and Poedts, 2004)

$$
\begin{aligned}
\nabla \cdot(\rho \boldsymbol{v}) & =0, \\
\rho(\boldsymbol{v} \cdot \nabla) \boldsymbol{v} & =\boldsymbol{j} \times \boldsymbol{B}-\nabla p, \\
E_{\mathrm{z}}+v_{\mathrm{x}} B_{\mathrm{y}}-v_{\mathrm{y}} B_{\mathrm{x}} & =\eta j_{\mathrm{z}}, \\
\eta:=\boldsymbol{N} / j_{\mathrm{z}} & \\
\nabla \cdot \boldsymbol{v} & =0 \\
\nabla \times \boldsymbol{B} & =\mu_{0} \boldsymbol{j}, \\
\nabla \cdot \boldsymbol{B} & =0,
\end{aligned}
$$

where $N$ is any non-ideal term and defines the effective resistivity $\eta$ with $j_{\mathrm{z}} \neq 0$. The non-idealness should be understood as some kind of spatial variation of a non-classical "resistivity" that is not necessarily the "classical" anomalous resistivity, but generating an electric field in that system, comoving with the plasma. This resistivity should enable the plasma to cross magnetic separatrices in a usually ideal MHD plasma. It cannot be expected that any macroscopic mechanism, depending on the macroscopic MHD parameters, can generate 
such a non-idealness or resistivity. As known, in solar flare reconnections, the resistivity needs to be much greater than that of classical Coulomb collisions, therefore the anomalous resistivity is considered. This anomalous resistivity expresses interactions of current-carrying electrons with electrostatic and electromagnetic waves generated in flare plasmas, e.g. by electric current instabilities. However, also the external electron beam can produce these waves. In both cases, it is natural to expect that at the saturation state of these waves their energy level as well as corresponding resistivity will be varying in space, i.e. having somewhere its maxima and minima (see e.g. Karlický and Jungwirth, 1989 or Karlický and Bárta, 2008). Therefore the resistivity is not a given property of the fluid, but a self-interaction of the whole plasmaelectromagnetic field-system. As will be shown in the following, just the locations of these extreme values of the resistivity and their very close vicinity are locations where we search for reconnection solutions. Our analysis is valid for every resistivity-model or non-idealness. We generate a local and necessary condition for $\boldsymbol{v}, \boldsymbol{B}$ and $\eta$.

Due to stationary Maxwell equations the electric field in the 2-D case has to be constant, i.e. $E_{\mathrm{z}}=$ const (see, e.g. Titov et al., 2004). To satisfy one necessary condition for magnetic reconnection, we have to assume $E_{\mathrm{z}} \neq 0$. We will later on see that we are able to separate reconnective annihilation solutions from (complete) reconnection solutions. We also analyse the resistive MHD system (9)-(15) for the limiting case $E_{\mathrm{Z}} \rightarrow 0$ or the asymptotical boundary condition $E_{\mathrm{Z}}=0$, i.e. $\boldsymbol{v}_{\infty} \times \boldsymbol{B}_{\infty}=0$ in the asymptotical, ideal region. The asymptotic boundary condition $\mathbf{v}_{\infty} \| \boldsymbol{B}_{\infty}$ enhances the probability that the stationary flows are stable, at least in the ideal region (see, e.g. Hameiri, 1998). The assumption that $E_{\mathrm{Z}}=0$, or better to say $E_{\mathrm{Z}} \rightarrow 0$, does not alter the results. This shows the boundary between nonideal reconnective flows and non-ideal but nonreconnective flows.

Another constraint in addition to the boundary conditions is the fact that the resistivity should fulfill the condition $\eta j_{\mathrm{Z}}^{2}>0$ or shorter $\eta>0$ to allow for positive dissipation only. We will later on see that this restricts the "allowed" plasma flows severely.

We calculate almost the complete solution space of incompressible resistive or non-ideal MHD close to the null points of flow field and magnetic field. The aim is to find the general correlation between the Jacobians of the plasma velocity and the magnetic field, and the shape of the Taylor-expanded resistivity.

A similar method to determine the structure of the non-ideal term and the flow and the magnetic field has been proposed and done, but for the case $j_{\mathrm{Z}}=0$ and for "global" fields, in the frame of a toy model in Nickeler and Fahr (2005).

In Sect. 3 we concentrate on the linearized fields and take only first order terms of the spatial variables into account. But basically the solutions found here could be also regarded as an exact solution when the density is completely constant, and velocity field and magnetic field are completely linear.

With this linear or linearized fields we can draw conclusions with respect to the other MHD quantities, like pressure, density and resistivity: The lowest order of the magnetic field is linear, the current density is constant, and therefore the Lorentz force $\boldsymbol{j} \times \boldsymbol{B}$ is linear in $x$ and $y$. This is the reason that all other terms should be also at the most of first order in the spatial variables. This leads to the conclusion that the plasma pressure $p$ is at highest order quadratic in $x$ and $y$, to allow for a linear pressure force, i.e. we can express $p$ by

$p=p_{0}+p_{1} x+p_{2} y+p_{3} x y+p_{4} x^{2}+p_{5} y^{2}$,

where $p_{0}$ to $p_{5}$ are constant coefficients.

As the velocity close to the stagnation point can be represented as a linear term in $x$ and $y$, the $\boldsymbol{v} \cdot \boldsymbol{\nabla v}$ term is also linear in $x$ and $y$. That means that all terms in the Euler equation are of first order, implying therefore that the mass density, $\rho$, has to be constant in lowest order close to the stagnation point. This holds even more for completely linear velocity, magnetic field and quadratic pressure.

Taking into consideration that at the magnetic null point $E_{\mathrm{Z}}$ equals $\eta j_{\mathrm{Z}}$ and if we assume that the resistivity is constant, then $E_{\mathrm{z}}=\eta j_{\mathrm{z}}$ everywhere in the vicinity of the neutral point. This implies that the term $v_{\mathrm{x}} B_{\mathrm{y}}-v_{\mathrm{y}} B_{\mathrm{x}}$ equals to zero within the linear region and therefore the flow is field-aligned everywhere within this region in lowest order of the spatial variables. In addition, for our asymptotical boundary conditions it implies that the resistivity must be zero around the null point. The same holds for special cases of non-constant resistivity, e.g. if $\eta$ is a function of the current density $j_{z}$ only, one can conclude that in the case of a linear field or in the vicinity of the null point the current density is constant and therefore also the function $\eta=$ const, as $\eta=\eta\left(j_{\mathrm{Z}}=\right.$ const $)=$ const.

To avoid such trivial solutions, namely field-aligned flows, which are non-reconnective in lowest order, the non-ideal term cannot be chosen at wish to enable the required topological classes of both, or all three vector fields $\boldsymbol{v}, \boldsymbol{B}$ and $\nabla \eta$. Therefore, to enable reconnection in the vicinity of the null point, the resistivity cannot be a function of the current density only. We have to regard the resistivity in resistive Ohm's law as a spatially dependent resistivity, i.e. $\eta=\eta(x, y)$. Such spatially dependent resistivities have also been used in similiar manner, e.g. by Al-Hachami and Pontin (2010), Hornig and Priest (2003) and Wyper and Jain (2011) ${ }^{1}$. We designate the function $\eta=\eta(x, y)$ as an "effective" resistivity or short resistivity. As the current density is constant inside the region of the linear field approximation, the resistivity is a substitute expression for a general non-ideal term. The magnetic field line structure, the structure of the stream lines and the structure of the non-ideal (resistive) term determine each other! The plasma should of course be able to cross the magnetic

\footnotetext{
${ }^{1}$ In 2-D such a method has not been applied up to now
} 
separatrices also in the region of constant current ${ }^{2}$, therefore a non-constant $\eta$ is necessary for magnetic reconnection close to the null point.

Written with all coefficients and comparing with all orders of $x$ and $y$, we get the following system of equations from the Euler or momentum equation

$$
\begin{aligned}
\rho\left(V_{11}^{2}+V_{12} V_{21}\right)+2 p_{4}-2 a j_{z} & =0, \\
\rho\left(V_{11}^{2}+V_{12} V_{21}\right)+2 p_{5}-2 b j_{z} & =0, \\
p_{3} & =0, \\
p_{1}=p_{2} & =0 .
\end{aligned}
$$

The Eqs. (17), (18), and (19) are of first order in $x$ and $y$, while Eq. (20) is of zeroth order.

\section{Solutions}

The general solution of the system (17)-(20) is now represented by

$$
\begin{aligned}
& p_{4}=-\frac{\rho}{2}\left(V_{11}^{2}+V_{12} V_{21}\right)+a j_{\mathrm{z}} \\
& p_{5}=-\frac{\rho}{2}\left(V_{11}^{2}+V_{12} V_{21}\right)+b j_{\mathrm{z}},
\end{aligned}
$$

with, as it seems, free parameters $V_{11}$ and $V_{12}$ and, of course, the "magnetic parameters" threshold current $j_{t}$ and actual current $j_{z}$. To guarantee a spontaneous process, i.e. a process where energy is only dissipated, it is necessary that $E_{\text {convective }} \cdot j_{z} \equiv\left(E_{\mathrm{Z}}+\boldsymbol{v} \times \boldsymbol{B}\right) \cdot j_{\mathrm{z}} \boldsymbol{e}_{\mathrm{z}}>0$. In the following we will therefore see that the parameters $V_{i j}$ are not all free, as they are determined by the fact that the quadric, representing the effective resistivity, must be an elliptic paraboloid (necessary critereon). We will therefore have to introduce another parameter $s$ to express $V_{21}$ as function of $V_{12}, V_{11}$ and $s$, as we will see in the following. The elliptic paraboloid is the only quadric that allows a positive resistivity with one zero, the vertex of the paraboloid, at the magnetic null point in the limiting case $E_{\mathrm{Z}} \rightarrow 0$. This implies necessarily a minimum for the resistivity in the limiting case. For the case $E_{\mathrm{z}} \neq 0$ the nullpoint of the vector fields can either be a minimum, or also a maximum.

All other quadrics can be excluded, with the exception of the degenerated case of a parabolic cylinder (cylindrical paraboloid) (see, e.g. Bartsch, 1984). The quadric $f(x, y, \eta)=0$, we analyse, is given by the resistive Ohm's law Eq. (11)

$$
\begin{aligned}
f(x, y, \eta)= & -\frac{E_{\mathrm{z}}}{j_{\mathrm{z}}}+\frac{2 a V_{11}}{j_{\mathrm{z}}} x^{2}+\frac{2 a V_{12}+2 b V_{21}}{j_{\mathrm{z}}} x y \\
& -\frac{2 b V_{11}}{j_{z}} y^{2}+\eta \equiv-\frac{E_{\mathrm{z}}}{j_{\mathrm{z}}}-\eta_{0}(x, y)+\eta,
\end{aligned}
$$

\footnotetext{
${ }^{2}$ namely the region inside the current sheet, where the maximum of the current is located
}

where the last part of the equation defines the spatially varying part of the resistivity $\eta_{0}$. Starting point is the fact that there are four invariants of any quadric (see, e.g. Bartsch, 1984; Bronstein, 1987). The corresponding invariants are given by:

I.

$$
\begin{aligned}
\Delta & =\left|\begin{array}{cccc}
2 a V_{11} / j_{\mathrm{z}} & \left(a V_{12}+b V_{21}\right) / j_{\mathrm{z}} & 0 & 0 \\
\left(a V_{12}+b V_{21}\right) / j_{z} & -2 b V_{11} / j_{\mathrm{Z}} & 0 & 0 \\
0 & 0 & 0 & \frac{1}{2} \\
0 & 0 & \frac{1}{2}-E_{\mathrm{z}} / j_{\mathrm{Z}}
\end{array}\right| \\
& =\left[4 a b V_{11}^{2}+\left(a V_{12}+b V_{21}\right)^{2}\right] / j_{\mathrm{Z}}^{2}
\end{aligned}
$$

II.

$$
\delta=\left|\begin{array}{ccc}
2 a V_{11} / j_{\mathrm{Z}} & \left(a V_{12}+b V_{21}\right) / j_{\mathrm{Z}} & 0 \\
\left(a V_{12}+b V_{21}\right) / j_{\mathrm{Z}} & -2 b V_{11} / j_{\mathrm{z}} & 0 \\
0 & 0 & 0
\end{array}\right|=0
$$

III.

$$
\begin{aligned}
t= & \left|\begin{array}{cc}
2 a V_{11} / j_{z} & \left(a V_{12}+b V_{21}\right) / j_{\mathrm{z}} \\
\left(a V_{12}+b V_{21}\right) / j_{z} & -2 b V_{11} / j_{\mathrm{z}}
\end{array}\right|+\left|\begin{array}{cc}
-2 b V_{11} / j_{\mathrm{z}} & 0 \\
0 & 0
\end{array}\right| \\
& +\left|\begin{array}{ll}
0 & 0 \\
0 & 2 a V_{11} / j_{\mathrm{z}}
\end{array}\right| \\
& =-\left[4 a b V_{11}^{2}+\left(a V_{12}+b V_{21}\right)^{2}\right] / j_{\mathrm{z}}^{2}
\end{aligned}
$$

IV.

$s=\operatorname{trace}(\delta)=2 V_{11}(a-b) / j_{\mathrm{z}}$.

The condition

$\Delta<0$ and $t>0$

is necessary and sufficient for getting an elliptic paraboloid, and obviously this can be fulfilled, as $\Delta=-t$. By using the Eq. (8) for substituting the expression for $a$ and $b$ and by using one of the inequalities in Eq. (29), the general solution can then be parameterized by the parameters $V_{11}, V_{12}, s$

$$
V_{21}=\frac{j_{\mathrm{t}}+j_{\mathrm{z}}}{j_{\mathrm{t}}-j_{\mathrm{z}}} V_{12}+2 s V_{11} \frac{\sqrt{j_{\mathrm{t}}^{2}-j_{\mathrm{z}}^{2}}}{j_{\mathrm{t}}-j_{\mathrm{z}}},
$$


with the restriction $s \in[-1,1]$. The cylindrical paraboloids are limiting cases with $s= \pm 1$, as is shown in the example in Fig. 2 (here the specific value is $s=-1$ ). Multiplication of Eq. (30) with $V_{12}$, inserting this expression into the expression for the eigenvalue $\lambda_{V}^{2}=V_{11}^{2}+V_{12} V_{21}$, and completing the square gives

$$
\begin{aligned}
\lambda_{V}^{2} & =V_{11}^{2}+V_{12} V_{21} \\
& =\left(V_{11}+s V_{12} \frac{\sqrt{j_{\mathrm{t}}^{2}-j_{\mathrm{Z}}^{2}}}{j_{t}-j_{\mathrm{z}}}\right)^{2}+\left(1-s^{2}\right) V_{12}^{2} \frac{j_{t}+j_{\mathrm{z}}}{j_{t}-j_{\mathrm{z}}} \geq 0 .
\end{aligned}
$$

If $\left|j_{\mathrm{z}}\right|<j_{\mathrm{t}}$ and $|s|<1$ are assumed, the magnetic field is of hyperbolic type and it is guaranteed that the stagnation point is also of hyperbolic type, i.e. has two purely real eigenvalues, namely one positive value and a negative counterpart. As mentioned already in the introduction, we proved here that also "typical" stagnation point flows are possible (" $X$ type"), and exclude here the discussion of "shear-like" flows.

Let us investigate now the geometry, respectively the slopes of the separatrices of the flow and the magnetic field. Defining $J_{\mathrm{Z}}:=j_{\mathrm{z}} / j_{\mathrm{t}}$, the magnetic separatrix is given by $A=0$ and $\left.A=\frac{\mu_{0}}{4}\left[\left(j_{t}-j_{\mathrm{z}}\right) y^{2}-\left(j_{\mathrm{t}}+j_{\mathrm{z}}\right) x^{2}\right)\right]$ (we restrict this to $\left.\left|J_{Z}\right|<1\right)$ and can therefore be expressed by

$$
\begin{gathered}
\sqrt{1-J_{\mathrm{z}}} y \pm \sqrt{1+J_{\mathrm{z}}} x=0 \\
\Leftrightarrow y= \pm \frac{\sqrt{1+J_{\mathrm{z}}}}{\sqrt{1-J_{\mathrm{z}}}} x:= \pm K_{\mathrm{m}} x,
\end{gathered}
$$

where $\pm K_{\mathrm{m}}$ is the slope of the both magnetic separatrix lines or to say the both asymptotes. As $V_{21}$ is given by the general solution, Eq. (30), by defining a stream function $\psi$ via

$v_{\mathrm{x}}=\frac{\partial \psi}{\partial y} \quad$ and $\quad v_{\mathrm{y}}=-\frac{\partial \psi}{\partial x}$,

we can integrate the above equations and get for the stream function

$\psi=\frac{V_{12}^{2}}{2} y^{2}-\frac{V_{21}^{2}}{2} x^{2}+V_{11} x y$.

The fluid separatrix is here given by $\psi=0$, geometrically these are asymptotes.

We will briefly discuss the problem that the magnetic separatrix is partially identical with the hydrodynamic separatrix. In this case the plasma flow can only take place across one part of the separatrix, or both separatrix lines are identical and no reconnection can take place.

One can clearly recognize that in the case $V_{11}=0$ and thus $\left(V_{21} / V_{12}\right)^{2}=\left(1+J_{\mathrm{Z}}\right) /\left(1-J_{\mathrm{z}}\right)$, both corresponding asymtotical branches (= separatrix lines) have the same slope. Thus the hydrodynamical separatrix and the magnetic separatrix are identical, the plasma cannot cross the magnetic separatrix and therefore no reconnection can take place.

Writing $y(x)=K_{V} x$ (if $V_{11}, V_{12} \neq 0$ ), where $K_{V}$ is the slope of the hydrodynamic separatrix, and inserting this into
$\frac{V_{12}^{2}}{2} y^{2}-\frac{V_{21}^{2}}{2} x^{2}+V_{11} x y=0$ with the parametric expression for $V_{21}$ in Eq. (30), we get the slopes $K_{V 1, V 2}$ of the two asymptotes/separatrix lines

$$
\begin{aligned}
K_{V 1,2} & =-\frac{V_{11}}{V_{12}} \pm \sqrt{\frac{V_{11}^{2}+V_{12} V_{21}}{V_{12}^{2}}} \\
& =\frac{-V_{11} \pm \sqrt{V_{11}^{2}+2 s V_{11} V_{12} \frac{\sqrt{1-J_{\mathrm{z}}^{2}}}{1-J_{\mathrm{Z}}}+V_{12}^{2} \frac{1+J_{\mathrm{z}}}{1-J_{\mathrm{Z}}}}}{V_{12}} \\
& =-V_{\text {rel }} \pm \sqrt{V_{\text {rel }}^{2}+2 s V_{\text {rel }} \frac{\sqrt{1-J_{\mathrm{Z}}^{2}}}{1-J_{\mathrm{z}}}+\frac{1+J_{\mathrm{z}}}{1-J_{\mathrm{z}}}},
\end{aligned}
$$

where $V_{\text {rel }}=V_{11} / V_{12}$. For $V_{12}=0 \neq V_{11}$ the asymptotes are given by $x=0$ and $y=s \sqrt{\left(1+J_{\mathrm{Z}}\right) /\left(1-J_{\mathrm{Z}}\right)} x$, and for $V_{12}=$ $V_{21}=0$ we get $x=0$ and $y=0$ as separatrices.

Let $V_{11}=V_{12}(=1)$, then

$$
\begin{aligned}
K_{V 1,2} & =-1 \pm \sqrt{\left(1+s \frac{\sqrt{1-J_{\mathrm{Z}}^{2}}}{1-J_{\mathrm{Z}}}\right)^{2}} \\
& =-1 \pm\left(1+s \frac{\sqrt{1-J_{\mathrm{Z}}^{2}}}{1-J_{\mathrm{Z}}}\right) .
\end{aligned}
$$

There is one corresponding slope of the flow separatrix with respect to one of the magnetic separatrix lines if $s= \pm 1$.

Therefore we can formulate the following theorem (analogous to known anti-reconnection theorems), which is now restricted to resistive dissipation only, i.e. vanishing nonresistive dissipation mechanisms.

\section{Theorem}

If the flow close to the null point is in good approximation incompressible, and other kinds of dissipation mechanisms than the resistive dissipation are negligible, then the plasma flow cannot cross the magnetic separatrices if for the both slopes of the both magnetic separatrix lines $K_{\mathrm{m}}=K_{\mathrm{m} 1}=$ $-K_{\mathrm{m} 2}$ with $K_{\mathrm{m}}=\sqrt{\left(1+J_{\mathrm{z}}\right) /\left(1-J_{\mathrm{z}}\right)}$ and the following is valid:

$$
\text { I. a) } K_{\mathrm{m}}=K_{V 1} \wedge \quad \text { b) }-K_{\mathrm{m}}=K_{V 2}
$$

or

$$
\text { II. a) } \left.K_{\mathrm{m}}=K_{V 2} \wedge \quad b\right)-K_{\mathrm{m}}=K_{V 1} \text {. }
$$

If only I.a) or I.b) or II.a) or II.b) is valid, then only one magnetic separatrix can be crossed, and as the other stream lines converge to the second magnetic separatrix line without crossings, one could call this reconnective annihilation (see, e.g. Priest and Forbes, 2000). The necessary condition for a complete non-crossing is $V_{11}=0$ for $V_{12} \neq 0$ and for only partly crossing flows $s= \pm 1$.

For almost all values of $s$, here $s=0$, the flow in Fig. 2 (left column) crosses all four separatrix branches (all two separatrix lines), the resistivity $\eta_{0}$ is positive (only zero at the 
null point, middle panel, the minimum resistivity case $)^{3}$, and their isocontours are ellipses (bottom panel). For the special parameter $s=-1$, it can be seen in Fig. 2 (right column) that the flow, like for the aforementioned magnetic reconnective annihilation solutions, crosses only the separatrix line with the positive slope, while it converges to the other magnetic separatrix, being almost field aligned (top panel), the resistivity $\eta_{0}$ being also positive (middle panel), and the isocontours of the resistivity are straight lines (bottom panel).

We now focus on the effective resistivity and therefore on the nature of ohmic heating, i.e. dissipation. As the resistivity is given by

$\eta=\frac{E_{z}}{j_{z}}+\eta_{0}$

we can now rewrite $\eta_{0}$

$\eta_{0}=\frac{\mu_{0}}{2} \frac{V_{11}}{j_{\mathrm{z}}}\left[\left(j_{t}+j_{\mathrm{z}}\right) x^{2}-2 s \sqrt{j_{\mathrm{t}}^{2}-j_{\mathrm{Z}}^{2}} x y+\left(j_{\mathrm{t}}-j_{\mathrm{z}}\right) y^{2}\right]$.

To ensure that the dissipation is positive, we prove that the term $\eta_{0}=\left(j_{\mathrm{t}}+j_{\mathrm{z}}\right) x^{2}-2 s \sqrt{j_{\mathrm{t}}^{2}-j_{\mathrm{z}}^{2}} x y+\left(j_{\mathrm{t}}-j_{\mathrm{z}}\right) y^{2}$ is larger than zero (or zero) for all $s$ and $x, y$. Let $K_{1,2}=j_{\mathrm{t}} \pm j_{\mathrm{z}}$ and $K_{1}, K_{2}>0$ as required for an X-point. Then

$$
\begin{aligned}
0 & \leq\left(\sqrt{K_{1}} x-s \sqrt{K_{2}} y\right)^{2} \\
& =K_{1} x^{2}-2 s \sqrt{K_{1} K_{2}} x y+s^{2} K_{2} y^{2} \\
& \leq K_{1} x^{2}-2 s \sqrt{K_{1} K_{2}} x y+K_{2} y^{2} .
\end{aligned}
$$

This implies that the sign of the resistivity $\eta$ depends on the sign of $\frac{V_{11}}{j_{z}}$. If the resistivity $\eta$ should have a maximum at the null point, the values $V_{11}$ and $j_{\mathrm{Z}}$ must have a different sign; in the case of minimum of $\eta$ the parameters must have the same sign. The first case seems to be the more "physical" or interesting case. The negativity of $\eta_{0}$ implies that the linear model is only valid in some small domain, in which $\eta>0$. In the next paragraphs we will discuss this problem in detail. As the dissipation should be positive, i.e. $\eta>0$, the values $V_{11}$ and $j_{\mathrm{Z}}$ must have the same sign in the case of a vanishing electric field $E_{\mathrm{Z}} \rightarrow 0$ in the ideal region.

For $s= \pm 1$, it can be recognized from Eq. (41) and from the bottom panel Fig. 2 (for the case $s=-1$ ) that the resistivity contour lines are straight lines given by $\eta_{0}=$ $\mu_{0} V_{11} /\left(2 j_{\mathrm{z}}\right)\left(\sqrt{j_{\mathrm{t}}+j_{\mathrm{z}}} x \pm \sqrt{j_{\mathrm{t}}-j_{\mathrm{z}}} y\right)^{2}$, and therefore the resistivity has the structure of a one-dimensional sheet. The case $s= \pm 1$ therefore reflects the 1-D character of the nonidealness, which can also be found, e.g. in the papers of Craig and Henton (1995), Tassi et al. (2002), Titov et al. (2004),

\footnotetext{
${ }^{3}$ Only the sign of spatially varying part of the resistivity $\eta_{0}$, depending on $V_{11} / j_{z}$, can change. This analysis does not change, i.e. neither the topology/geometry of the the isocontours of streamlines and magnetic field lines, nor the isocontours of the resistivity are changed.
}
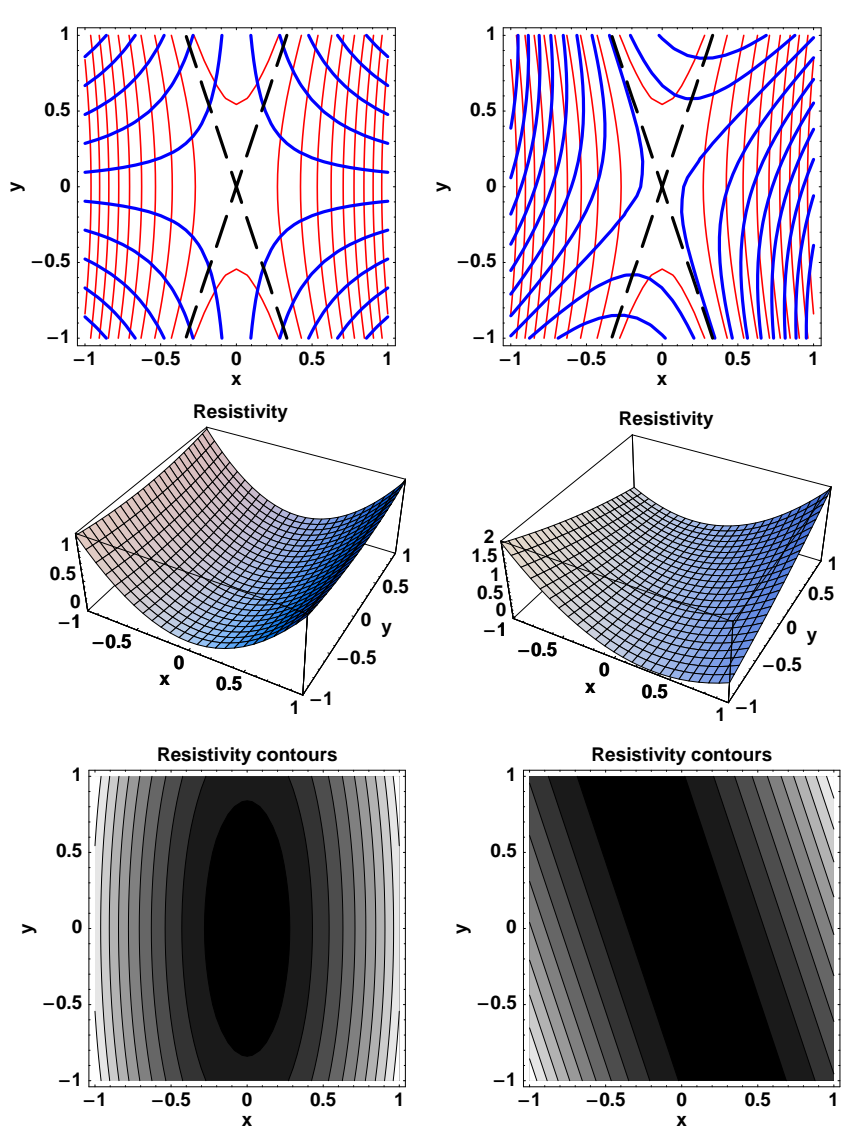

Fig. 2. Flow lines are thick (blue), thin (red) lines are magnetic field lines, thick (black) dashed lines are magnetic separatrix lines (top panels). Flow for the parameters $V_{11}=V_{12}=1$ and $s=0$ (left column), crossing all four magnetic separatrix branches (left top panel), and $s=-1$ (right column), flow lines crossing only two magnetic separatrix branches (right top panel), shape of the positive resistivity $\eta_{0}$ (middle panels), and the elliptic shaped contours of the resistivity (bottom panels).

and Watson and Craig (1998). This implies that for complete crossing of all separatrices, a 2-D shape of the non-ideal term is necessary.

If the electric field is non-zero, we have to distinguish two cases:

1. $V_{11} / j_{Z}>0$ the resistivity is positive definite, (as $E_{\mathrm{z}} / j_{\mathrm{z}}>0$ everywhere), the elliptic paraboloid is directed upwards and represents therefore a local minimum of the resistivity, or

2. $V_{11} / j_{\mathrm{Z}}<0\left(E_{\mathrm{Z}} / j_{\mathrm{z}}>0\right.$ everywhere $)$ the case that the resistivity-paraboloid in this case is directed upside down, and represents therefore a maximum with a shift depending on $E_{\mathrm{z}}$ :

$$
\eta(x, y)=E_{\mathrm{z}} / j_{\mathrm{Z}}+\eta_{0}(x, y)
$$

As $\eta$ has a maximum, the validity of the local model is of course given by the ellipse $\eta=0$. Outside this ellipse the 
resistivity is not defined, as it represents only the lowest order parts of a Taylor expansion. Formally the resistivity is getting negative, as the elliptic paraboloid touches the $\eta=0$ plane. With the help of the principal axis transformation, one can calculate the small axis of the ellipse (a), and the large axis (b)

$$
\begin{aligned}
& \text { (a) } l_{1}^{2}=-\frac{2 E_{\mathrm{z}} / j_{\mathrm{Z}}}{\mu_{0} V_{11} \frac{j_{t}}{j_{\mathrm{z}}}-\left|\mu_{0} V_{11}\right| \sqrt{1-s^{2}+s^{2} \frac{j_{\mathrm{t}}^{2}}{j_{\mathrm{Z}}^{2}}}} \\
& \text { (b) } l_{2}^{2}=-\frac{2 E_{\mathrm{Z}} / j_{\mathrm{Z}}}{\mu_{0} V_{11} \frac{j_{\mathrm{t}}}{j_{\mathrm{z}}}+\left|\mu_{0} V_{11}\right| \sqrt{1-s^{2}+s^{2} \frac{j_{\mathrm{t}}^{2}}{j_{\mathrm{Z}}^{2}}}}
\end{aligned}
$$

In the case that $s \rightarrow \pm 1$, we get the case of reconnective annihilation with the large axis $l_{2} \rightarrow \infty$. Due to the "infinite" axis the flow can only cross one part of the magnetic separatrix, as can be seen in the bottom panel of Fig. 2 (for the case $s=-1$ ). The minimum value of the smallest axis, representing the scale on which the linear field approximation can be valid, is then given by $l<l_{1, \min }=E_{\mathrm{z}} /\left(\mu_{0}\left|V_{11}\right| j_{\mathrm{t}}\right)$.

\section{Discussion and conclusions}

We analyse the system of stationary, slightly resistive/nonideal magnetohydrodynamics (MHD) in the vicinity of singular points of flow and field to determine the boundary between reconnection solutions and non-reconnective solutions. This is an important point, because not every non-ideal term or resistivity guarantees a reconnection process (see, e.g. Schindler et al., 1988 or Hornig and Schindler, 1996).

To consider structurally stable fields in $2-\mathrm{D}$, so-called "generic fields", it is necessary that the corresponding stream and flux functions are of Morse type, i.e. the corresponding vector fields in the vicinity of null points must depend linearly on the coordinates. This implies that the non-ideal or resistive term is a quadric in the vicinity of the null point. Thus our mathematical proofs in this manuscript have to be regarded as a tool for analysing all generic solutions with a small non-idealness around the null point. It gives the necessary conditions for all structurally stable solutions in the vicinity of the null point.

Reconnection is defined as a process where plasma crosses magnetic boundaries (separatrices) and shows a constant electric field in the invariant direction $\left(E_{\mathrm{z}}=\right.$ const $)$. We proved that crossing of magnetic separatrices requires also an X-type stagnation point flow if the effective resistivity and thus the dissipation should be positive. The existing analytical solutions, done for constant resistivity, show a structure called reconnective annihilation: these solutions allow the plasma flow only to cross one of the two separatrix lines. We proved mathematically that this property of the plasma flow is connected with the one-dimensional structure of the nonidealness. We also proved that spatial structure of the resistivity or non-idealness must be two-dimensional to allow the plasma to cross both separatrix lines. If the non-idealness has a one-dimensional, sheet-like structure, the plasma flow can only cross one part of the separatrix, similar to well known magnetic reconnective annihilation solutions. Therefore, our mathematical model proves and explains well the properties of analytical reconnective annihilation solutions of Craig and Henton (1995), Tassi et al. (2002), Titov et al. (2004), and Watson and Craig (1998). Our model delivers a necessary condition for all existing 2-D models of magnetic reconnection.

The analyses started by us here will be extended to configurations that have a saddle point of the resistivity. This extension will maybe provide us with a deviation from pure (X-type) stagnation point flow.

Acknowledgements. D. H. Nickeler acknowledges financial support from GAAV ČR grant No. IAA300030804 and M. Karlický from GA ČR grant No. 300030701.

Topical Editor R. Forsyth thanks three anonymous referees for their help in evaluating this paper.

\section{References}

Al-Hachami, A. K. and Pontin, D. I.: Magnetic reconnection at 3D null points: effect of magnetic field asymmetry, aap, 512, 1-10, 2010.

Amann, H.: Gewöhnliche Differentialgleichungen, Walter de Gruyter, Berlin, 1995.

Arnold, V.: Ordinary Differential Equations, Springer, Berlin, 1992.

Bartsch, E.: Mathematische Formeln, 250-258, VEB Fachbuchverlag, Leipzig, 1984.

Baty, H., Forbes, T. G., and Priest, E. R.: Petschek reconnection with a nonlocalized resistivity, Phys. Plasmas, 16, 012102012102-5, 2009.

Bronstein, I. N. and Semendjajew, K. A.: Taschenbuch der Mathematik, Verlag Harri Deutsch, Frankfurt/Main, 1987.

Bruce, J. W. and Giblin, P. J.: Curves and singularities, Cambridge University Press, 1992.

Cowley, S. W. H.: Comments on the merging of nonantiparallel magnetic fields, J. Geophys. Res., 81, 3455-3458, 1976.

Craig, I. J. D. and Henton, S. M.: Exact solution for steady state incompressible magnetic reconnection, ApJ, 450, 280-288, 1995.

Craig, I. J. D. and Rickard, G. J.: Linear models of steady state, incompressible magnetic reconnection, A\&A, 287, 261-267, 1994.

Goedbloed, J. P. and Poedts, S.: Principles of Magnetohydrodynamics, Cambridge University Press, Cambridge, 2004.

Hameiri, E.: Variational principles for equilibrium states with plasma flow, Phys. Plasmas, 5, 3270-3281, 1998.

Hornig, G. and Priest, E.: Evolution of magnetic flux in an isolated reconnection process, Phys. Plasmas, 10, 2712-2721, 2003.

Hornig, G. and Schindler, K.: Magnetic topology and the problem of its invariant definition, Phys. Plasmas, 3, 781-791, 1996.

Karlický, M. and Bárta, M.-J.: Fragmentation of the Current Sheet, Anomalous Resistivity, and Acceleration of Particles Solar Phys., 247, 335-342, 2008. 
Karlický, M. and Jungwirth, K.: Solar flares and the dynamics of Langmuir waves in current-carrying plasmas, Solar Phys., 124, 319-338, 1989.

Nickeler, D. and Fahr, H.-J.: Reconnection at the heliopause, Adv. Space Res., 35, 2067-2072, 2005.

Nickeler, D., Goedbloed, H., and Fahr, H.-J.: Stationary fieldaligned MHD flows at astropauses and in astrotails. Principles of a counterflow configuration between a stellar wind and its interstellar medium wind, aap, 454, 797-810, 2006.

Parnell, C., Neukirch, T., and Priest, E.: The structure of threedimensional magnetic neutral points, Phys. of Plasmas, 3, 759770, 1996.

Peixoto, M. M.: On structural stability, Ann. Math., 69, 199-222, 1959.

Petschek, H. E.: The Physics of Solar Flares, edited by: Hess, W. N., AAS-NASA Symp., 425-439, 1964.

Priest, E. and Cowley, S. W. H.: Some comments on magnetic field reconnection, J. Plasma Phys., 14, 271-282, 1975.

Priest, E. and Forbes, T.: Magnetic Reconnection, Cambridge University Press, Cambridge, 2000.

Priest, E., Titov, V. S., Vekstein, G. E., and Rikard, G. J.: Steady linear X-point magnetic reconnection, J. Geophys. Res., 99, $21467-$ 21480, 1994.

Reitmann, V.: Reguläre und chaotische Dynamik, B.G. Teubner Verlagsgesellschaft, Leipzig, 1996.

Schindler, K., Hesse, M., and Birn, J.: General magnetic reconnection, parallel electric fields, and helicity, J. Geophys. Res., 93, 5547-5557, 1988.
Sonnerup, B. U. Ö.: Solar Systems Plasma Physics, edited by: Lanzerotti, L. J., Kennel, C. F., and Parker, E. N., vol. 3, North Holland, Amsterdam, pp. 45, 1979.

Sonnerup, B. U. Ö., Baum, P. J., Birn, J., Cowley, S. W. H., Forbes, T. G., Hassam, A. B., Kahler, S. W., Matthaeus, W. H., Park, W., Paschmann, G., Priest, E. R., Russell, C. T., Spicer, D. S., and Stenzel, R.: Solar Terrestrial Physics: Present and Future, edited by: Butler, D. M. and Papadopoulos, K., NASA, Ref. Publ. Vol. 1120, 1-1-1-71, 1984.

Sweet, P. A.: The production of high-energy particles in solar flares Nuovo Cimento Suppl., 8, 188-196, 1958.

Tassi, E., Titov, V. S., and Hornig, G.: Exact solutions for magnetic annihilation in curvilinear geometry, Phys. Lett. A, 302, 313317, 2002.

Titov, V. S. and Hornig, G.: Magnetohydrodynamic flows sustaining stationary magnetic nulls, Phys. Plasmas, 7, 3542-3550, 2000.

Titov, V. S., Tassi, E., and Hornig, G.: Exact solutions for steady reconnective annihilation revisited Phys. Plasmas, 11, 4662-4671, 2004.

Vasyliunas, V. M.: Theoretical models of magnetic field line merging. I., Rev. Geophys., 13, 303-336, 1975.

Watson, P. G. and Craig, I. J. D.: Magnetic Annihilation and Reconnection in Two Dimensions, ApJ, 505, 363-368, 1998.

Wyper, P. F. and Jain, R.: Torsional magnetic reconnection: The effects of localizing the non-ideal $(\eta \boldsymbol{J})$ term, J. Plasma Phys., 77, 843-855, 2011. 